\title{
Research on the Optimization and Ascension of Target Accuracy for RF Systems
}

\author{
LI Ying ${ }^{1, \text { a }}$ \\ ${ }^{1}$ State Key Laboratory of UAV Special Technology, Northwestern Polytechnical University, Xi'an \\ Shaanxi, 710072, P.R.China \\ aliying315@126.com
}

\begin{abstract}
Keywords: Optimization and Ascension, RF Systems, Simulation Experiment
\end{abstract}
\begin{abstract}
A digital built-in calibration (BIC) system is presented to automatically adjust the linearity performance of a tunable RF low-noise amplifier (LNA) operating at $2.4 \mathrm{GHz}$. In this paper, we conduct research on optimization and ascension of target accuracy for RF systems. To minimize the area and power overhead of the system, an envelope detector was employed to reduce the speed of operation for the ADC and digital blocks, and an efficient IM3 estimation method has been developed. Closed-loop simulations of this calibration system and a low-noise amplifier with digitally-tunable third-order linearity performance showed the feasibility of the synthesized digital blocks in standard CMOS technology. The experimental result proves the effectiveness of proposed method.
\end{abstract}

\section{Introduction}

Testing and parameter tuning after fabrication are becoming inevitable to improve the quality and yield of integrated circuits in CMOS technologies, especially in analog or RF circuit designs that are more sensitive to performance degradations from aging effects and process variations. Built-in calibration (BIC) is a kind of method, allowed to improve reliability and to increase the lifetime of analog/rf circuit. Is one of the benefits of BIC system, they can restrict the manufacturing cost reduce reliance on external testing equipment and the required number of input/output pins. For example, a standard linear measurement of RF circuit, such as low noise amplifier (LNA) including third-order estimate intercept point (IIP3) based on multiple input third-order intermodulation distortion measurement (IM3). In the traditional way to do this, the test tone is applied to measure the frequency domain intermodulation distortion using high-performance equipment such as spectrum analyzer. However, RF integrated circuit test of high sampling rate gigahertz range is expensive. Therefore, since the calibration technology requirements have been on the rise, because they allow external tool to capture to eliminate the high frequency test output signal. Analog designs with digitally-tunable capabilities for impedance matching, gain and frequency tuning of LNAs [1]-[2], second-order nonlinearity and mismatch correction for mixers [3], linearity enhancements for baseband filters [4] are some examples showing the relevance of incorporating digital techniques to improve the performance of analog circuits. Measurement of one of the important parameters such as gain and linear is able to assess the performance of the circuit under test (reduce) based on the different frequency spectrum characteristics. As a result, more and more need efficient on-chip digital resources on the chip spectrum characteristics and digital correction control. Toward this goal, a spectrum analyzer, etc. [5] on a chip design BIC and built-in self-test (bist). Alternative methods of reaction related circuit of the envelope. For example, in an envelope detector [6], analog-to-digital converter (ADC), and digital signal processor is used for the calibration. In [7-13] in addition, it puts forward the evaluation of the output spectrum of the envelope detector output connected to reduce the extraction of the third order distortion, which requires a single chip implementation of FFT engine.

BIC design system, one of the major challenges is to reduce the additional circuit, while maintaining sufficient accuracy. In this study, the numerical control self-calibration in figure 1 is developed IM3 estimate rf cutting and automatically adjust the best IM3 performance. [8] the low 
noise amplifier topology are used to demonstrate the function of the BIC system is put forward. It includes the digital switches allow IM3 tuning. Minimize BIC on the piece of hardware system, the use of some design techniques. The envelope detector as frequency conversion technology, we used FFT engine design method from the [9] area - efficient spectral analysis.

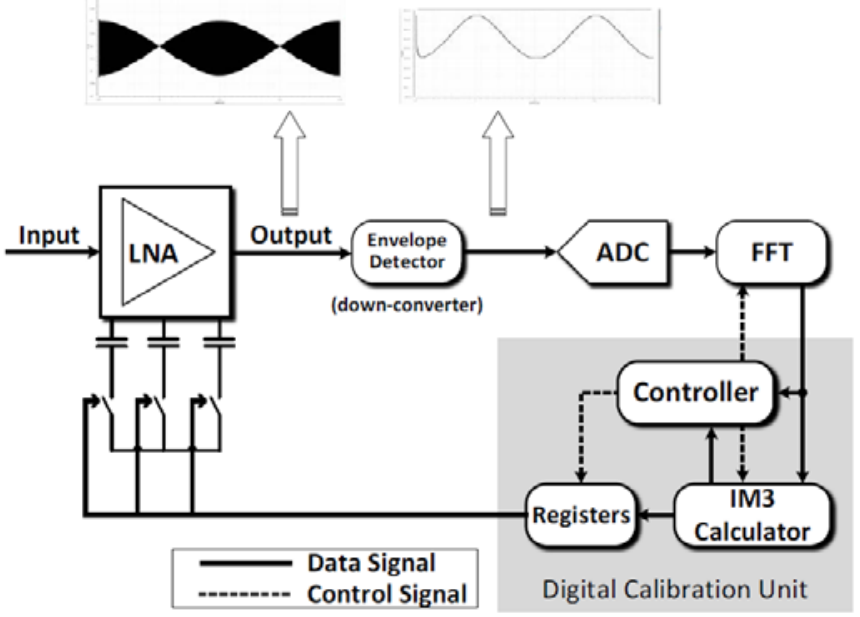

Figure 1: The Block Diagram of the Built-in Calibration (BIC) Scheme

The interaction between magnetic fields and the nu- clear/electronic magnetic moments represents a flexible tool for the control of the internal and external degrees of freedom in atoms or molecules, widely employed in precision measurements, frequency metrology, and coherent manipulations of quantum systems. In frequency metrology, the presence of magnetic fields may represent a limit on the realization of specific targets. In the attractive context of the "magic" wavelength combining a vanishing differential shift of the clock levels with the Lamb-Dicke regime greatly reducing the motional effects [1-3], the quest for "magic" magnetic field values where first order Zeeman shift and magnetic fluctuation of the atomic transition is annulled, was proposed in [4] and studied experimentally in rubidium [5]. In the following sections, we will discuss the issues in detail.

\section{The System Design}

The Tunable Subthreshold Low-Noise Amplifier and Envelope Detector. The Fig. 2 shows the schematic of the cascode LNA with inductive source degeneration from our previous work, in which this subthreshold LNA with linearity enhancement was proposed [8].

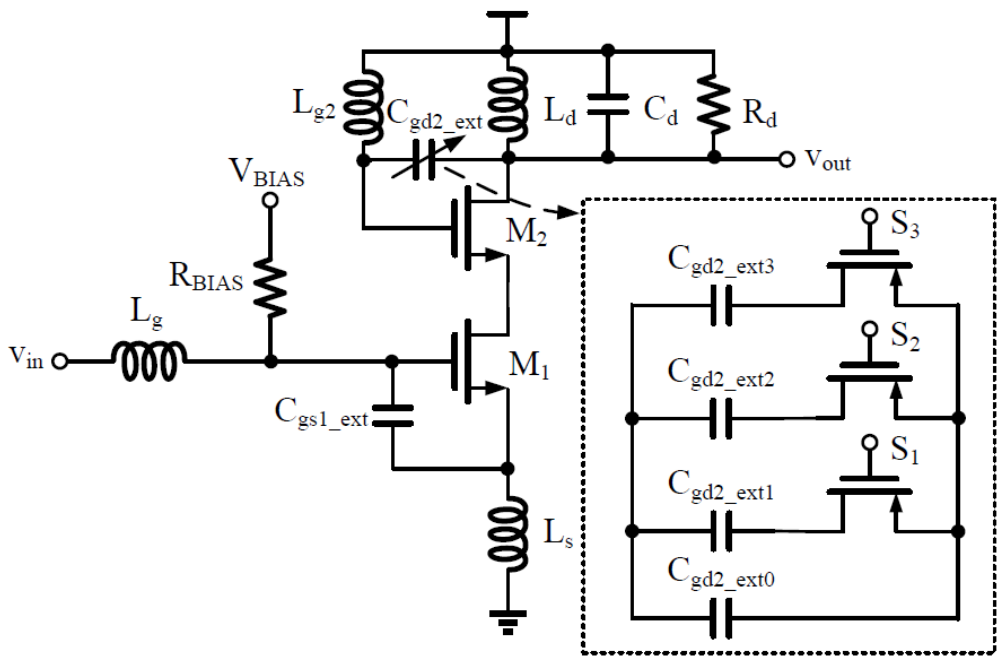

Figure 2: The Low-noise amplifier with digitally-tunable linearity 


\begin{tabular}{ccccc}
\hline Code $\left[\mathbf{S}_{3} \mathbf{S}_{2} \mathbf{S}_{1}\right]$ & $\mathbf{S}_{11}[\mathbf{d B}]$ & $\mathbf{S}_{21}[\mathbf{d B}]$ & $\mathbf{N F}[\mathbf{d B}]$ & IM3 $[\mathbf{d B c}]$ \\
\hline 000 & -29.5 & 12.9 & 4.42 & 33.33 \\
001 & -26.1 & 12.9 & 4.41 & 32.57 \\
010 & -23.9 & 12.8 & 4.39 & 31.89 \\
011 & -22.2 & 12.6 & 4.39 & 31.21 \\
100 & -28.2 & 11.7 & 4.53 & 35.57 \\
101 & -32.9 & 12.2 & 4.53 & 35.49 \\
110 & -39.8 & 12.4 & 4.50 & 34.70 \\
111 & -37.5 & 12.7 & 4.50 & 34.11 \\
\hline
\end{tabular}

Figure 3: The Simulation Data

The transmitter consists of pseudorandom bit sequence generators, which generate bit sequence, which is fed to coder to produce an electrical coded signal. The pulses, after passing through a low pass filter are then modulated with continuous wave (CW) laser radiation of wavelength 1550nm to obtain optical pulses .Mach Zender Modulator is used as external modulator to obtain optical pulses from electrically coded signal. PRBS has launched 10Gbps rate. Analysis of NRZ and RZ code encoder by using the influence of different modulation schemes is vital. Here the use of the light source is a continuous wave laser with the Lorenz center frequency of emission of the 193.4thz center, power emission wavelengths were 1dB 1547-1560 CW. Electronic low-pass filter using the Bessel filter. Optical fiber section consists of single mode fibers and dispersion compensated fibers in the channel.

The Calibration Architecture. Fig. 4 displays a block diagram of the proposed calibration method. To estimate the IM3 components of the LNA, a two-tone input signal is applied and the output signal is down-converted through an envelope detector. The down-conversion avoids the use of an ADC with a high sampling frequency, which would be challenging to design and would consume excessive power. Despite the envelope detector also creates the nonlinear, estimate the best low noise amplifier IM3 no significant impact on performance, because the same closed-loop correction for envelope detector nonlinear under the condition of low noise amplifier IM3 setting changes to determine the optimal output. Output signal in frequency domain by ADC after digital envelope analysis using the FFT engine and a clock frequency is equal to the ADC sampling frequency. Digital calibration device IM3 IM3 calculator estimates of the magnitude of the plural FFT output. Once IM3 estimation process of a cycle is completed, the controller block changes the capacitor array to repeat the adjustment switch amplifier before IM3 IM3 assessment process. Before each cycle of output than IM3 calculator output and smaller values (show better IM3 performance) is stored in a register as the best condition. The different combination of the process is iterative a n a capacitor array within the low noise amplifier. Control block and other digital block is suitable for the best combination of capacitor array is complete the tuning process has been completed.

The cancelation of the first order Zeeman effect is produced by the atomic dressing at a rf frequency much larger than the effective Larmor precession, equivalent to a frequency modulation of the nuclear magnetization and a shielded nuclear response to the static magnetic field. The different rf response for the ground and excited states of the clock transition leads to crossing nodes in the energy diagram, where the atoms become non- magnetic. This change from a paramagnetic system to an non-magnetic one shares a strong analogy with a Landau theory of phase transition. In addition at magic static field values the rf dressing engineers a Zeeman-insensitive atomic clock. This magic cancelation arises from the non-linear magnetic Hamiltonian associated to the rf dressing of the two-electron system. Even if the dressing does not eliminate the second-order Zeeman contribution, its contribution to clock state separation is strongly decreased by an operation at a magic magnetic field. The stability of the ratio between rf dressing amplitude and rf angular frequency required to produce a target non-magnetic state matched to the aimed optical clock accuracy is experimentally reachable. The present approach of a magic magnetic field cannot be applied to tensorial ac shifts. 


\section{The Experimental Simulation}

Set-up and Structure of Experiment. The Fig. 4 visualizes a two-tone test for the characterization of an RF circuit (CUT) with an envelope detector to down-convert high-frequency signals to low frequencies.
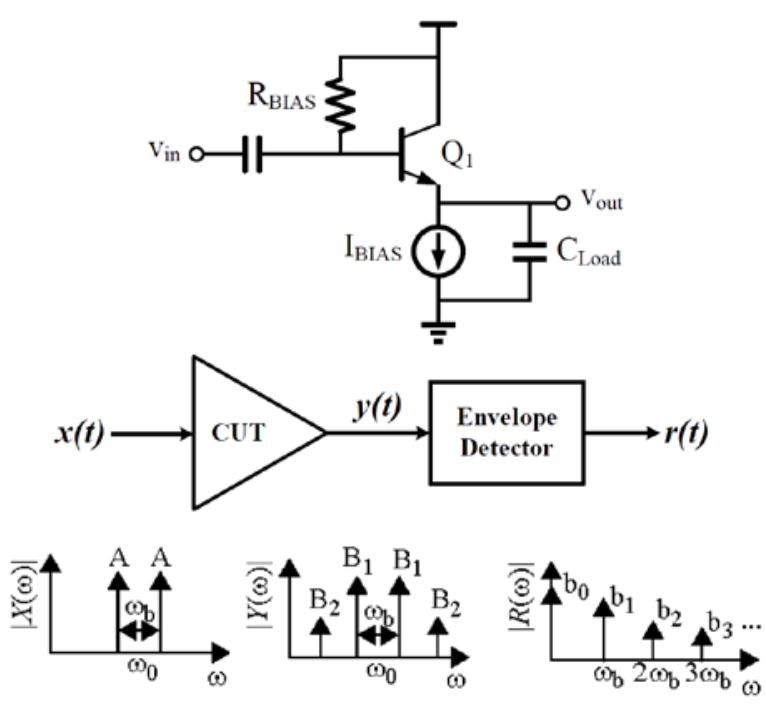

Fig.4: The Simulation Set-up

Higher values, more easily manageable in the laboratory, are simply obtained by increasing the rf frequency and applying the scaling. The accuracy of the dressed energy and the magic pairs strongly depends on the atomic parameters as tested by a numerical evaluation of the continued fraction. A complete "full-scale" calculations as reported in $[4,9]$ would be required for accurate magic numerical values including all the digits recommended for a correct evaluation. To highlight the resolution which should be targeted for canceling the Zeeman shifts below the 10-19 level, we have used a 6 digit resolution for figures when necessary.

The Simulation Result One. In this section, results are reported that demonstrate the proposed linearity calibration technique for the example LNA using the analog/mixed-mode simulation (AMS) environment in Cadence to assess the analog circuits and digital blocks (implemented with Verilog-HDL). Thus, the IM3 components appear at $2.399 \mathrm{GHz}$ and $2.402 \mathrm{GHz}$. The $1 \mathrm{MHz}$ tone spacing results in low-frequency envelope detector output components with $1 \mathrm{MHz}$ spacing.
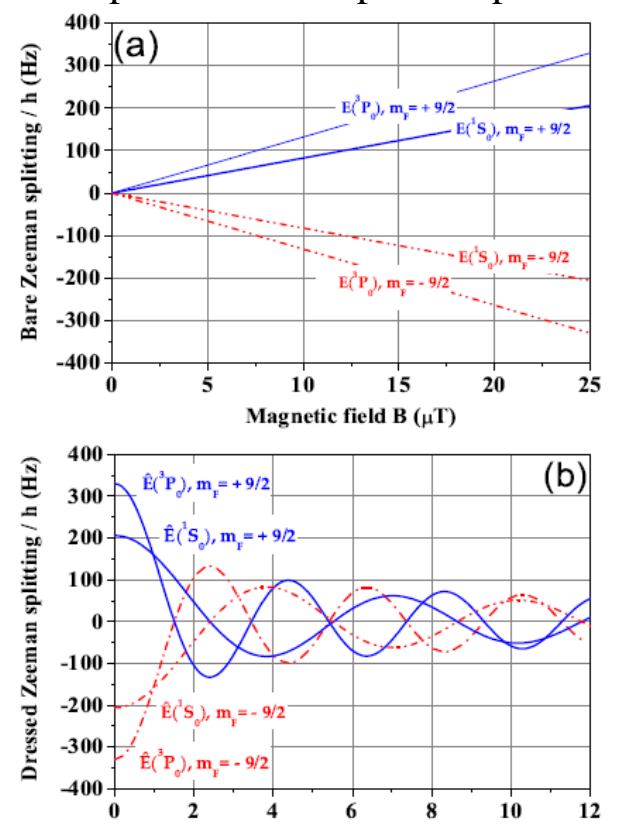

Figure 5: The Simulation Result Set One 
The Simulation Result Two.The output initialization and each of the capacitor combinations are shown in the figure. /Best_IM3 and /SW_BEST present the best IM3 component value and the corresponding capacitor switch array status, respectively. Also, the /IM3 and /SW_CODE data buses show the current IM3 component value and the corresponding capacitor switch array. The best code is [100] and IM3 component value is $35.57 \mathrm{dBc}$, which is the optimum value among all the cases. This optimum setting can be identified from the minimum "estimated IM3" number in the figure, which is the value of the /IM3 variable in Fig. 6 (converted to decimal form, where the fractional bits are dropped since the number is large). If a system-on-chip already includes an onchip FFT and ADC, then the FFT can be used for processing outputs from multiple CUTs and envelope detector. The digital calibration blocks can be set to powered down mode in order to save power consumption when this one-time calibration process is not needed. Hence, the system is best suited for start-up calibrations to compensate for process variations as well as for period calibrations when the CUT is idle. It represents the magnitude of the third-order intermodulation component extracted by the IM3 calculator. The total simulation time is $485 \mu \mathrm{s}$, which includes delays of $1.2 \mu \mathrm{s}$ to allow settling of the LNA output after capacitor array changes.
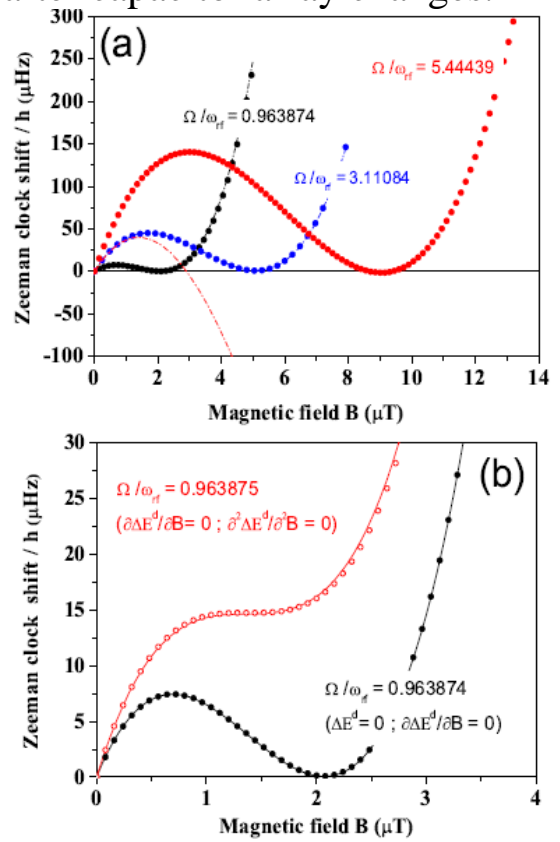

Figure 6: The Simulation Result Set Two

\section{Conclusions}

A built-in calibration system for performance tuning of RF amplifiers was presented. This architecture makes the on-chip calibration of linear optimization estimation IM3 in two-tone test. Reduce the area and the overhead of the power system, an envelope detector is used to reduce the operation speed of the ADC and digital blocks, and an effective IM3 estimation method. This calibration system closed-loop simulation and low noise amplifier digitally - tunable third-order linear performance show the feasibility of the synthetic standard digital CMOS technology. In conclusion, the proposed calibration system for RF amplifier linearity tuning is effective. In the future, we plan to use more research to modify our framework.

\section{References}

[1] F. Ahmad and R.K. Bullough, J. Phys. B: Atom. Molec. Phys. 7, L275 (1974).

[2] H.-C. Choi, Y.-J. Kim, S.-W. Yoo, S.-Y. Hwang, and S.-H. Lee, “A programmable 0.8-V 10-bit 60-MS/s 19.2-mW 0.13- $\mu$ m CMOS ADC operating down to 0.5 V," IEEE Trans. Circuits Syst. II, Exp. Briefs, vol. 55, no. 4, pp. 319-323, Apr. 2008. 
[3] M. Barragan, R. Fiorelli, D. Vazquez, A. Rueda, and J. L. Huertas, "On-chip characterisation of RF systems based on envelope response analysis,” Electron. Lett.,vol. 46, no. 1, pp. 36-38, Jan. 2010.

[4] Dai, Yitang, et al. "Performance Improvement in Analog Photonics Link Incorporating Digital Post-Compensation and Low Noise Electrical Amplifier." (2014): 1-1.

[5] Labidi, Tarek, et al. "Optical Phase Sensitive Amplification for Microwave Photonics Applications: intermodulation distortion analysis." International Conference on Fibre Optics and Photonics. Optical Society of America, 2014.

[6] A. Valdes-Garcia, F.-L. Hussien, J. Silva-Martinez, and E. Sanchez-Sinencio, “An integrated frequency response characterization system with a digital interface for analog testing," IEEE J. Solid-State Circuits, vol. 41, no. 10, pp. 2301-2313, Oct. 2006.

[7] Cunha, G. C. L., et al. "An FPGA-based digital predistorter for RF power amplifier linearization using cross-memory polynomial model." Integrated Nonlinear Microwave and Millimetre-wave Circuits (INMMiC), 2014 International Workshop on. IEEE, 2014.

[8] D. Han, B. S. Kim, and A. Chatterjee, "DSP-driven self-tuning of RF circuits for processinduced performance variability,” IEEE Trans. Very Large Scale Integr. (VLSI) Syst., vol. 18, no. 2, pp. 305-314, Feb. 2010.

[9] J.-Y. Ryu, B. Kim, and I. Sylla, "A new low-cost RF built-in self-test measurement for systemon-chip transceivers,” IEEE Trans. Instrum. Meas., vol. 55, no. 2, pp. 381-388, Apr. 2006.

[10] H.-K. Cha, S.-S. Song, H.-T. Kim, and K. Lee, "A CMOS harmonic rejection mixer with mismatch calibration circuitry for digital TV tuner applications,” IEEE Micro. Wirel. Compon. Lett., vol. 18, no. 9, pp. 617-619, Sep. 2008.

[11] Chen, Kenle, et al. "Highly Linear and Highly Efficient Dual-Carrier Power Amplifier Based on Low-Loss RF Carrier Combiner." IEEE TRANSACTIONS ON MICROWAVE THEORY AND TECHNIQUES (2014).

[12] M. Mobarak, M. Onabajo, J. Silva-Martinez, and E. Sanchez-Sinencio, "Attenuationpredistortion linearization of CMOS OTAs with digital correction of process variations in OTA-C filter applications,” IEEE J. Solid-State Circuits, vol. 45, no. 2, pp. 351-367, Feb. 2010.

[13] Kheirkhahi, Alireza, et al. "Improved Envelope Injection and Termination (EIT) RF Power Amplifier With Envelope Equalization for Mobile Terminal Applications." Microwave Theory and Techniques, IEEE Transactions on 62.2 (2014): 341-351. 